\title{
Blastobotrys adeninivorans and $B$. raffinosifermentans, two sibling yeast species which accumulate lipids at elevated temperatures and from diverse sugars
}

Stéphane Thomas ${ }^{1 \dagger}$, Daniel R. A. Sanya ${ }^{1 \dagger}$, Florian Fouchard ${ }^{1}$, Huu-Vang Nguyen ${ }^{1}$, Gotthard Kunze², Cécile Neuvéglise ${ }^{1}$ and Anne-Marie Crutz-Le Coq ${ }^{1 *}$ (1)

\begin{abstract}
Background: In the context of sustainable development, yeast are one class of microorganisms foreseen for the production of oil from diverse renewable feedstocks, in particular those that do not compete with the food supply. However, their use in bulk production, such as for the production of biodiesel, is still not cost effective, partly due to the possible poor use of desired substrates or poor robustness in the practical bioconversion process. We investigated the natural capacity of Blastobotrys adeninivorans, a yeast already used in biotechnology, to store lipids under different conditions.

Results: The genotyping of seven strains showed the species to actually be composed of two different groups, one that (including the well-known strain LS3) could be reassigned to Blastobotrys raffinosifermentans. We showed that, under nitrogen limitation, strains of both species can synthesize lipids to over $20 \%$ of their dry-cell weight during shake-flask cultivation in glucose or xylose medium for $96 \mathrm{~h}$. In addition, organic acids were excreted into the medium. LS3, our best lipid-producing strain, could also accumulate lipids from exogenous oleic acid, up to $38.1 \pm 1.6 \%$ of its dry-cell weight, and synthesize lipids from various sugar substrates, up to $36.6 \pm 0.5 \%$ when growing in cellobiose. Both species, represented by LS3 and CBS $8244^{\top}$, could grow with little filamentation in the lipogenic medium from 28 to $45^{\circ} \mathrm{C}$ and reached lipid titers ranging from $1.76 \pm 0.28$ to $3.08 \pm 0.49 \mathrm{~g} / \mathrm{L}$ in flasks. Under these conditions, the maximum bioconversion yield $\left(Y_{F A / S}=0.093 \pm 0.017\right)$ was obtained with $L S 3$ at $37^{\circ} \mathrm{C}$. The presence of genes for predicted subunits of an ATP citrate lyase in the genome of LS3 reinforces its oleaginous character.

Conclusions: Blastobotrys adeninivorans and B. raffinosifermentans, which are known to be xerotolerant and genetically-tractable, are promising biotechnological yeasts of the Saccharomycotina that could be further developed through genetic engineering for the production of microbial oil. To our knowledge, this is the first report of efficient lipid storage in yeast when cultivated at a temperature above $40^{\circ} \mathrm{C}$. This paves the way to help reducing costs through consolidated bioprocessing.
\end{abstract}

Keywords: Microbial oil, Lipid metabolism, Biotechnology, Saccharomycotina, Thermotolerance, Oleaginous yeasts

\footnotetext{
*Correspondence: anne-marie.le-coq@inra.fr

†'Stéphane Thomas and Daniel R. A. Sanya contributed equally to this

work

1 Micalis Institute, INRA, AgroParisTech, Université Paris-Saclay,

78350 Jouy-en-Josas, France

Full list of author information is available at the end of the article
}

(c) The Author(s) 2019. This article is distributed under the terms of the Creative Commons Attribution 4.0 International License (http://creativecommons.org/licenses/by/4.0/), which permits unrestricted use, distribution, and reproduction in any medium, provided you give appropriate credit to the original author(s) and the source, provide a link to the Creative Commons license, and indicate if changes were made. The Creative Commons Public Domain Dedication waiver (http://creativecommons.org/ publicdomain/zero/1.0/) applies to the data made available in this article, unless otherwise stated. 


\section{Background}

The use of renewable feedstocks to produce microbial oil for biodiesel or other applications has become a scientific and environmental issue over the last two decades. Oleaginous yeasts, defined as those that can accumulate lipids to over $20 \%$ of their dry-cell weight (DCW), are still being screened and can be found in different taxa. A useful criterion is their ability to grow on pentoses or polysaccharides, which are lignocellulosic sugars. However, although some of these yeasts are immediately suitable for providing useful enzymes (such as sugar hydrolases), their capacity to be custom engineered for specific lipid production will require additional effort. Conversely, known genetically-tractable yeasts may not have yet been tested for their oleaginous potential.

Blastobotrys adeninivorans is part of a basal clade of the Saccharomycotina subphylum [1] and diverged long before Saccharomyces cerevisiae. The species was first described in 1984, under the name of Trichosporon adeninovorans [2], and successively reclassified into the genera Arxula (adeninivorans) in 1990 [3] and Blastobotrys in 2007 [1]. A few natural isolates of soil, plant (wood hydrolysate, silage), or animal origin have been described in the literature or deposited in culture collections. Among them, the type strain CBS $8244^{\mathrm{T}}$ and the industrial strain LS3 have been the best studied, LS3 being used to develop auxotrophic recipients for genetic transformation $[4,5]$.

Since the early 1990s, the species has attracted attention because of its interesting biochemical, physiological, and genetic properties. First, it exhibits a versatile metabolism, indicative of the presence of various degradative enzymes and pathways linked to central metabolism [6]. One to several strains can assimilate a number of sugar substrates, such as D-galactose, D-xylose, L-arabinose, raffinose, sucrose, trehalose, cellobiose, starch, and arbutin, and can also ferment some of them (glucose, sucrose, and starch) [6, 7]. Organic acids (e.g., pyruvate, acetate, and butyrate), as well as aromatic compounds (e.g., hydroxybenzoates), may also serve as a growth source [6, 7]. Moreover, various nitrogenous compounds may serve as nitrogen sources (e.g., urea, formamide, ethanolamine, and most amino acids), both carbon and nitrogen sources (e.g., adenine, acetamide, uric acid, putrescine, and some amino acids) [6, 7], or an energy source via nitrate reductase [8]. Second, B. adeninivorans exhibits extremophilic traits, such as halotolerance, osmotolerance, and thermotolerance. This last characteristic is particularly interesting, as only a few yeasts, especially few biotechnological yeasts, have been found to grow at temperatures over $40{ }^{\circ} \mathrm{C}[9,10]$. Indeed, $B$. adeninivorans is able to grow at up to $48{ }^{\circ} \mathrm{C}$ [11] and survive a few hours at $55{ }^{\circ} \mathrm{C}$ [12]. In addition, as shown in the case of the LS3 strain, temperature can induce a reversible developmental state, with the cells being mainly in the yeast form at lower temperatures and (pseudo)hyphae forms at higher temperatures (above $42{ }^{\circ} \mathrm{C}$ ) [11]. Third, with an eye towards metabolic engineering, valuable information has been brought to light by sequencing of the complete genome of LS3 [13] and integrative plasmids and recipient strains for genetic engineering have been developed [14]. The small size of the haploid genome of this yeast $(11.8 \mathrm{Mb})$ is an additional advantage for gene function studies or chassis strain development for various applications.

Blastobotrys adeninivorans has earned its stripes as a biotechnological yeast mainly after being engineered in the laboratory of Kunze. It has been engineered for diverse applications, such as for a biosensor, a platform for protein expression, and a solventogenic yeast for butanol production [14]. Efficient cultivation in bioreactors has also been achieved, with Stöckmann et al. reporting a biomass titer of $240 \mathrm{~g} / \mathrm{L}$ under pressure [15]. The ability of $B$. adeninivorans to produce lipids has been rarely reported in the literature. Strain LS3 was included in an assay to monitor lipid bodies by impedance spectroscopy a decade ago [16]. More recently, Olstorpe et al. [17] reported the relative fatty acid (FA) composition of two strains of this species, without however mentioning the total amount of lipid.

Several yeast species are well-known lipid producers. Three have attracted most of the research efforts in recent years [18]: Yarrowia lipolytica and Lipomyces starkeyi, belonging to the Saccharomycotina, and Rhodotorula (Rhodosporidium) toruloides, belonging to the Basidiomycota. All three species exhibit a genome size of over $20 \mathrm{Mb}$ [19-21]. L. starkeyi and R. toruloides are known for their utilization of a wide range of substrates and their outstanding lipogenic capabilities (up to 70\% DCW as lipids) [22-24]. L. starkeyi is particularly well suited for rapid and simultaneous utilization of sugar mixtures, reaching a lipid content of $52 \%$ DCW and a lipid titer of $13.3 \mathrm{~g} / \mathrm{L}$ in a mixture of cellobiose and glucose [25]. Strain diversity or culture conditions may alter lipid production; to maximize lipid production, the synthesis of endopolysaccharides under nitrogen limitation should be avoided [26]. Although progress has been made in recent years, the genetic engineering of these yeasts is still a shortcoming [18]. B. adeninivorans is phylogenetically close to $Y$. lipolytica [27]. Some strains of Y. lipol$y$ tica, as well as other species in the genus, are naturally more prone to store lipids from exogenous hydrophobic compounds than to turn glucose into lipids. Lipid content can easily reach $30 \%$ DCW [28] in the former case and generally 6 to $15 \% \mathrm{DCW}$ in the latter [28, 29]. Metabolic engineering and/or process optimization, as well as strain screening, have been successfully used to increase 
lipid content above $35 \%$ DCW and reportedly up to $90 \%$. $Y$. lipolytica has also required genetic engineering or specific culture conditions to utilize xylose, cellobiose, or cellulose polymers, which are major components of lignocellulosic materials. Depending on the strain, biotechnological processes should be fine-tuned to prevent excessive excretion of citric acid, which competes with lipid production in the presence of excess carbon, and strong filamentation, which depends on multiple parameters (dissolved oxygen, medium composition, and initial carbon concentration, etc.) and may alter the process of lipid production and its yield [30, 31].

None of these species has been reported to grow at temperatures above $37^{\circ} \mathrm{C}$, though some thermotolerant strains may have been isolated. An $R$. toruloides DMKU3TK16 mutant obtained through an adaptive breeding strategy can store lipids at approximately 14\% DCW at $37^{\circ} \mathrm{C}$ [32]. However, working at upper temperatures could provide specific advantages for compound solubilization (e.g., FA), help to minimize the costs of cooling of bioreactors and facilitate consolidated bioprocesses. These processes are considered to be relevant at a temperature around $50{ }^{\circ} \mathrm{C}$ as microbial enzymes involved in the degradation of hemicellulose/cellulose or starch have been generally described to have optimal temperatures above $40^{\circ} \mathrm{C}$, up to $60^{\circ} \mathrm{C}[10,33,34]$.

The upper permissive temperature for growth of eucaryotes has been considered to be near $60^{\circ} \mathrm{C}$ [35], far below that of eubacteria and archae. Relatively few thermophilic fungi were found to grow at elevated temperatures, optimally around $55{ }^{\circ} \mathrm{C}$, and tolerated up to $61{ }^{\circ} \mathrm{C}[36,37]$. Some of these (not yeast-like) fungi might be useful bioconverters of lignocellulosic residues into sugars [37]. Yeast species comprising strains able to grow at temperatures up to $45^{\circ} \mathrm{C}$ were listed in a specific survey by Robert et al. in 2015 [9]. Among the 28 most thermotolerant species, two are well-known for different applications not related to lipid production, Ogataea (Hansenula) polymorpha and Kluyveromyces marxianus [38]. Few others were reported with a lipid content over $20 \%$ DCW in screening studies, such as Kodamaea ohmeri [39] and Kurtzmaniella cleridarum [40]. We are not aware of studies concerning lipid accumulation at high temperatures, except for $K$. marxianus. The potential of $K$. marxianus as an oleaginous yeast is unclear. A strain isolated from Kefir exhibited over 30\% DCW in lipids [41], while different strategies-including genetic modifications typically used in other organisms - to increase lipogenesis of several $K$. marxianus strains yielded at best a FA content of $16 \%$ DCW [42].

Because of its aforementioned advantageous traits, we reasoned that $B$. adeninivorans could be a valuable thermotolerant oleaginous yeast. Here, we assessed the ability of Blastobotrys species to naturally produce and accumulate lipids from a set of different substrates. Several strains of what was believed to be $B$. adeninivorans were used to examine the robustness of lipid production in the species. However, in the course of our study, strain genotyping showed that they can be divided into two different groups and various marker sequences actually placed the LS3 group into the Blastobotrys raffinosifermentans species. We took advantage of the thermotolerance of these yeasts and assayed lipid production at various temperatures, up to $45^{\circ} \mathrm{C}$. Overall, the natural capacity of these two species to produce lipids is promising and may be an alternative to currently-studied oleaginous yeasts.

\section{Results and discussion}

Nitrogen limitation and lipid accumulation in strain LS3

Oleaginous yeasts generally synthesize and abundantly store lipids in lipid droplets under specific environmental conditions, one of which is nitrogen limitation [43, 44 ]. In addition to FA synthesis, these yeasts can import and direct exogenous FAs into the lipid storage pathway, sometimes with particularly high efficiency, such as in $Y$. lipolytica [45].

We examined the capacity of strain LS3 to store lipids after neo-synthesis from glucose or after accumulation from exogenous oleic acid. We performed flask cultivation in YNB-based media with two different carbon sources at a fixed concentration of $30 \mathrm{~g} / \mathrm{L}$ (glucose or a mixture of oleic acid and glucose) and two different concentrations of $\mathrm{NH}_{4} \mathrm{Cl}$ as a unique nitrogen source $(5 \mathrm{~g} / \mathrm{L}$ or $0.75 \mathrm{~g} / \mathrm{L})$ to vary the carbon-to-nitrogen ratio $(\mathrm{C} / \mathrm{N})$. Efficient lipid storage occurred following growth in both substrates to above $30 \%$ DCW. However, as expected, it strongly relied on a low-nitrogen concentration when glucose was the sole substrate (Fig. 1a). When the exogenous FA C18:1 was provided, the FA profile of the strain was enriched in C18:1 (Fig. 1b), suggesting entry of the FA directly into the storage pathway. BODIPY staining showed storage to occur in the form of neutral lipids in lipid droplets in both cases (Fig. 1c). Our results show that strain LS3 has a good natural capacity for both lipogenesis and lipid accumulation upon FA uptake; in reaching approximately 30\% DCW as lipids from glucose, it may be qualified as oleaginous.

\section{The genome of strain LS3 encodes a putative ATP citrate lyase}

The complete genome sequence of LS3 (first identified as $B$. adeninivorans, then reassigned to B. raffinosifermentans; see below and Table 1), already recognized to be of biotechnological interest, was published a few years ago [13]. We searched the genome for potential "markers" of oleaginicity by mining the genome of LS3 for specific 

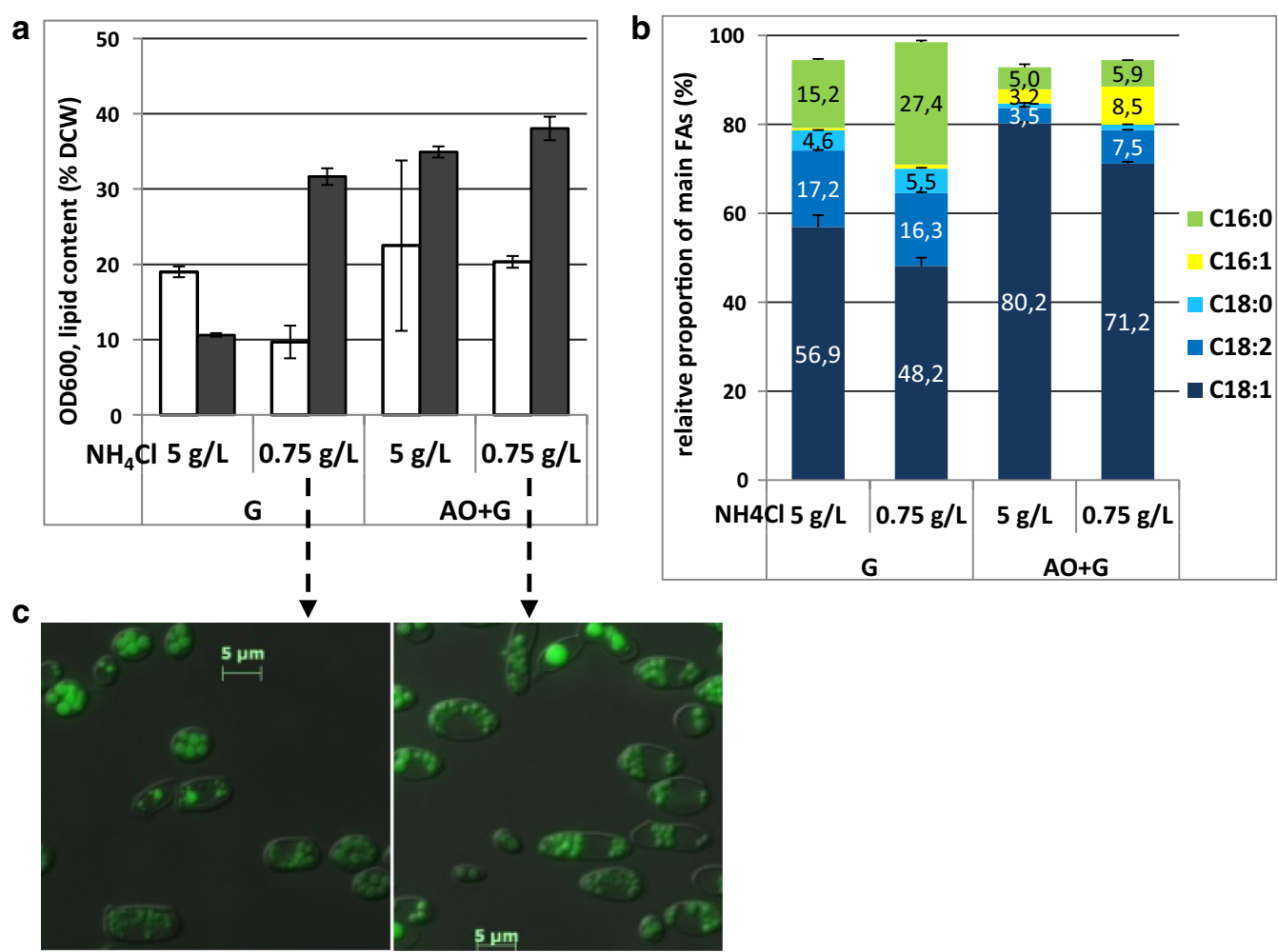

Fig. 1 Effect of limiting nitrogen on the synthesis and accumulation of lipids in strain LS3. LS3 was cultivated for $72 \mathrm{~h}$ in YNB-based medium with carbon source at $30 \mathrm{~g} / \mathrm{L}$. The concentrations of $5 \mathrm{~g} / \mathrm{L}$ and $0.75 \mathrm{~g} / \mathrm{L} \mathrm{NH}_{4} \mathrm{Cl}$ led to a $\mathrm{C} / \mathrm{N}$ ratio of 90 and 60 , respectively, for cultures in glucose (G) and to a C/N ratio of 16 and 106, respectively, for cultures in a mixture of oleic acid and glucose ( $A O+G)$. a Cell density is expressed as $O_{600}($ white bars) and total lipid content as the percentage of dry-cell weight (black bars). Average values and standard deviations $(n=2)$ are shown in the histograms. b Relative proportion (\%) of main FAs in each FA profile. c BODIPY-stained lipid droplets in cells sampled from low-nitrogen cultures in glucose (left) or in the presence of oleic acid (right)

Table 1 List of strains, received as Blastobotrys adeninivorans and reassigned to two different species

\begin{tabular}{|c|c|c|c|c|c|}
\hline Strain & Synonym & Isolated from & By & Reference & New species assignment \\
\hline CBS $8244^{\top}$ & CSIR 577, CLIB 1468 & Soil, The Netherlands (adenine-enrichment) & Middelhoven, 1983 & {$[2]$} & B. adeninivorans (type strain) \\
\hline CBS 7766 & & Reptile (liver and intestines), Sweden & Mattsson, 1993 & & B. adeninivorans \\
\hline CBS 7350 & & Maize silage, The Netherlands & Middelhoven, 1987 & & B. adeninivorans \\
\hline LS3 & & Wood hydrolysates, Russia (Siberia) & $\begin{array}{l}\text { Kapultsevich, selected } \\
\text { as industrial strain }\end{array}$ & {$[9]$} & B. raffinosifermentans \\
\hline CBS 8335 & & Soil (clay-like, pH 8.5), Italy & Middelhoven 1996 & & B. raffinosifermentans \\
\hline CBS 7370 & CSIR 1117 & Soil (humus-rich), South Africa & van der Walt & {$[3]$} & B. raffinosifermentans \\
\hline CBS 7377 & CSIR 1118 & Soil, South Africa & van der Walt, 1988 & {$[3]$} & B. raffinosifermentans \\
\hline
\end{tabular}

CSIR Council for Scientific and Industrial Research (Pretoria, South Africa), CLIB CIRM-Levures (France)

genes involved in lipid metabolism, using proteins of $Y$. lipolytica as a query (Table 2). First, cytoplasmic ATP citrate lyase (ACL), which can efficiently convert respiratory citrate to acetyl-CoA for FA biosynthesis, is considered to be a signature (first biochemical then genetic) of oleaginous yeasts [46-48]. ACL, of which the subunits are encoded by two distinct genes in LS3, could be confidently predicted by its high amino acid similarity with this enzyme in $Y$. lipolytica (Table 2). The presence of these genes suggests the native oleaginous character of the Blastobotrys strain. Second, we found that LS3 possesses a copy of an $A A L$-like gene encoding putative peroxisomal acyl-CoA synthetase (Table 2). Homologous gene products in $Y$. lipolytica, which has a family of ten genes of Acyl/Aryl-CoA-ligases (AAL), were shown to be responsible for the activation of FAs into acyl-CoA 
Table 2 Selected genes of lipid metabolism in the genome of B. raffinosifermentans LS3

\begin{tabular}{|c|c|c|c|c|}
\hline Gene identifier & 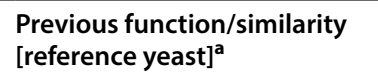 & New function prediction & $\begin{array}{l}\text { Amino acid \% identity } \\
\text { (coverage) }\end{array}$ & Process \\
\hline ARAD1B07414 & $\begin{array}{l}\text { Succinyl CoA ligase (alpha } \\
\text { subunit) [sc] }\end{array}$ & $\begin{array}{l}\text { ATP:citrate lyase (subunit) } \\
\text { EC:2.3.3.8 }\end{array}$ & 88\% (99\%) with YALIOE34793 & $\begin{array}{l}\text { Formation of cytosolic acetyl-CoA } \\
\text { precursor of FA synthesis }\end{array}$ \\
\hline ARAD1D32164 & $\begin{array}{l}\text { Succinyl CoA ligase (beta subu- } \\
\text { nit) [sc] }\end{array}$ & $\begin{array}{l}\text { ATP:citrate lyase (subunit) } \\
\text { EC:2.3.3.8 }\end{array}$ & $82 \%$ (100\%) with YALIOD24431 & $\begin{array}{l}\text { Formation of cytosolic acetyl-CoA } \\
\text { precursor of FA synthesis }\end{array}$ \\
\hline ARAD1C08250 & $\begin{array}{l}\text { Acyl-CoA:diacylglycerol acyl- } \\
\text { transferase (DGAT) [sc] }\end{array}$ & $\begin{array}{l}\text { DGAT (no change) } \\
\text { EC:2.3.1.20 }\end{array}$ & $69 \%$ (59\%) with YALIOE32769 & Formation of TG \\
\hline ARAD1D42460 & $\begin{array}{l}\text { Acyl-CoA:sterol acyltransferase } \\
{[\mathrm{sc}]}\end{array}$ & $\begin{array}{l}\text { DGAT } \\
\text { EC:2.3.1.20 }\end{array}$ & $42 \%$ (86\%) with YALIOD07986 & Formation of TG \\
\hline ARAD1C30118 & $\begin{array}{l}\text { Weakly similar to FAT2 peroxiso- } \\
\text { mal AMP binding protein [sc] }\end{array}$ & Acyl-CoA synthetase & $\begin{array}{l}\text { 38\% (99\%) with YALIOE12419 (10 } \\
\text { paralogs). PTS1 motif }\end{array}$ & $\begin{array}{l}\text { Formation of acyl-CoA (peroxiso- } \\
\text { mal activation of FA) }\end{array}$ \\
\hline
\end{tabular}

${ }^{a}$ In the current (first) version of the annotated genome of LS3 in GRYC database. First-round annotation was performed using BLAST against S. cerevisiae (sc) as the reference yeast to look for similarity

b $\mathrm{AKL}$ in $\mathrm{C}$ terminus of the protein which likely targets it to peroxisome

[48]. Although various types of acyl-CoA synthetases are found in yeast (e.g., Faa2 in S. cerevisiae), the distribution of these particular $A A L$-like genes was hypothesized to be biased towards oleaginous species [48]. Third, we examined the formation of triacylglycerol (TG). In yeast, TG synthesis relies on esterification of diacylglycerol with a third FA [49]. This last step of TG formation has been considered to be a limiting step for oil storage. It involves acyl-CoA dependent diacylglycerol acetyltransferases (DGAT) and/or phospholipid:diacylglycerol acetyltransferases (PDAT), which use phospholipids as FA donors. S. cerevisiae, Y. lipolytica [50], and B. raffinosifermentans LS3 (Table 2) all possess one gene encoding a PDAT enzyme. S. cerevisiae possesses only one DGAT gene, whereas Y. lipolytica $[51,52]$ and LS3 (Table 2) have two DGATs, possibly emphasizing their capacity for TG storage, a preferred form of oil storage in oleaginous yeasts. While our study was being conducted, the genetic content of strain CBS $8244^{\mathrm{T}}$ (type strain of B. adeninivorans) was analyzed in a patent in view of lipid production [53]. The genome reportedly contained the two genes for ACL and two different genes for DGAT, as in LS3. The genome sequence is not available, however.

\section{Diversity among strains and species assignment}

We continued our investigation of the oleaginous character of the species by expanding our investigation to several strains. Strains LS3 and CBS $8244^{\mathrm{T}}$ were previously suggested to belong to different genetic subgroups, given the size of their four chromosomes and DNA-fingerprints [54]. We thus genotyped five strains of various origins obtained from the CBS collection (Table 1) along with LS3 and CBS $8244^{\mathrm{T}}$.

Using AluI fingerprinting of intergenic spacer rDNA (IGS), we found the seven strains to be clearly distributed into two distinct groups, a first group of three strains, including CBS $8244^{\mathrm{T}}$, and a second group of four strains, including LS3 (Fig. 2a). The sequences of ITS and D1D2 showed the second group to be homogenous for this

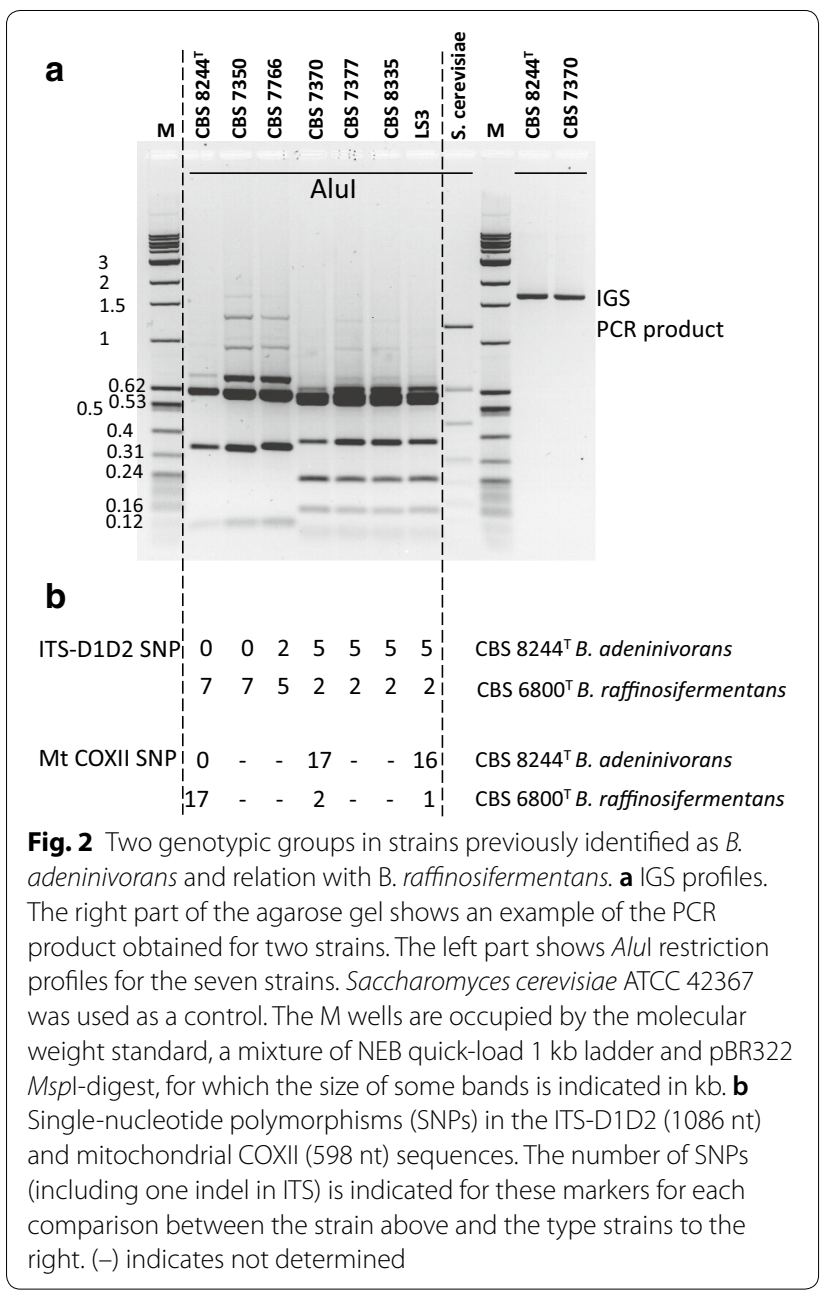


marker (no sequence polymorphism between strains), whereas it differs from the first group by three to five nucleotides (Fig. 2b, Additional file 1: Figure S1). The sequence of mtCOXII in CBS 7370 and LS3 confirmed the proximity of these strains, while showing divergence of CBS $8244^{\mathrm{T}}$ (Fig. 2b). The sequence of these markers for strains of the LS3 group better matched those of $B$. raffinosifermentans, according to the YeastIP database [55]. This species, closely related to $B$. adeninivorans, was described in 2007 and represented by a unique strain at that time [1]. Our results highlight the existence of two distinct groups among strains previously identified as $B$. adeninivorans, resulting in the reassignment of the LS3 group to the B. raffinosifermentans species.

\section{Capacity of sugar assimilation of B. raffinosifermentans and $B$. adeninivorans}

We examined the ability of the various strains to grow on three different simple carbohydrates, potentially useful as carbon and energy sources for microbial oil production. All strains exhibited relatively similar growth curves when cultivated in minimal medium supplemented with glucose or xylose, which reflects efficient assimilation of these substrates (maximum growth rate from 0.13 to $0.16 \mathrm{~h}^{-1}$ and stationary phase reached within 24 to $30 \mathrm{~h}$ in microplates). In contrast, glycerol promoted much slower and delayed growth, depending on the strain and substrate concentration (Additional file 1: Figure S2). For $B$. raffinosifermentans strains, the maximum growth rate ranged from 0.078 to $0.12 \mathrm{~h}^{-1}$ in $10 \mathrm{~g} / \mathrm{L}$ glycerol and 0.025 (CBS 8335) to $0.1 \mathrm{~h}^{-1}$ in $2 \mathrm{~g} / \mathrm{L}$ glycerol. This phase was generally preceded by a much slower growth phase or lag phase, especially in low concentrations of glycerol, extending up to $60 \mathrm{~h}$. For strains of the B. adeninivorans species (CBS $8244^{\mathrm{T}}$ group), growth was very weak $(10 \mathrm{~g} / \mathrm{L}$ glycerol) or not visible (2 g/L glycerol) after $96 \mathrm{~h}$ cultivation in microplates (Additional file 1: Figure S2). In plate assays with $10 \mathrm{~g} / \mathrm{L}$ glycerol, growth was also reproducibly observed to be delayed relative to growth on glucose, and all seven strains reached lower-density streaks on glycerol than glucose after 7 days at $28{ }^{\circ} \mathrm{C}$. As a control, $Y$. lipolytica W29 grew similarly on both glycerol and glucose plates (data not shown).

Glycerol assimilation is a typical variable trait among yeasts [56]. Glycerol is generally regarded to be an efficient carbon source for $L$. starkeyi [22] and even a preferred substrate for $Y$. lipolytica, especially as it is first consumed when both glycerol and glucose are present $[26,57]$. In contrast, most strains of S. cerevisiae do not grow in synthetic glycerol media without successful adaptation or medium supplementation with complex compounds, amino acids, or nucleobases [56]. Our results underline a difference in glycerol catabolism between some oleaginous yeasts and the two Blastobotrys species under study, or at least in its regulation. More favorable environmental conditions or conditions of possible adaptation are yet to be determined for efficient glycerol assimilation. However, glycerol is not only a potential substrate for growth but also the precursor backbone esterified by FAs in triglycerides. Sugar/glycerol blends or glycerol co-feeding have been successfully used to improve lipogenesis $[58,59]$ and would be interesting to evaluate in this particular context of poor glycerol catabolism, providing that transport would not be a limiting step.

\section{Capacity for lipogenesis of $B$. raffinosifermentans and $B$. adeninivorans}

We evaluated the robustness of lipogenesis among strains and investigated possible phenotypic diversity concerning this trait by assaying lipid production over time of two strains of each species (LS3 and CBS 7377 for one and CBS $8244^{\mathrm{T}}$ and CBS 7766 for the other). They were cultivated in glucose or xylose, in which all strains grew easily. We periodically measured the $\mathrm{OD}_{600}$ and FA content of the cells, as well as sugars and organic acids in the medium, throughout the 96-h cultivation in flasks under

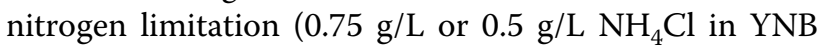
medium with $30 \mathrm{~g} / \mathrm{L}$ carbon source, leading to a mass carbon-to-nitrogen ratio $(\mathrm{C} / \mathrm{N})$ of 60 and 90 , respectively). Under these conditions, lipogenesis began at the start of growth for all strains. The highest amount of lipids in the cells was reached after $72 \mathrm{~h}$ and ranged from 14 to $29 \%$ DCW (Figs. 3, 4). Lipogenesis was negligibly better at $\mathrm{C} / \mathrm{N} 90$ than $\mathrm{C} / \mathrm{N} 60$ (up to $10 \%$ better) but it lowered growth and global sugar consumption.

The four strains exhibited different behaviors in terms of growth and metabolism, lipid accumulation, organic acid excretion, and sugar consumption. There were no strict species-specific correlations. CBS 7377 was the slowest and least efficient strain for lipid accumulation, whereas LS3 (of the same species) reached the highest lipid content. Conversely, the OD reached by LS3 in stationary phase was the lowest of all strains. However, different OD may reflect morphological differences between strains rather than differences in biomass production. The results of the strains of $B$. adeninivorans were more similar to each other. Overall, both species cultivated in low-nitrogen medium reached a lipid content above $20 \%$. Sugar uptake was more efficient for LS3 than for other strains in all media (Figs. 3, 4). With few exceptions, $<75 \%$ of the initial sugar was consumed by the end of experiment, leaving room for optimization of the medium and culture conditions. Within the time of the experiment, B. raffinosifermentans LS3 reached the highest lipid content among all four strains, whereas $B$. 

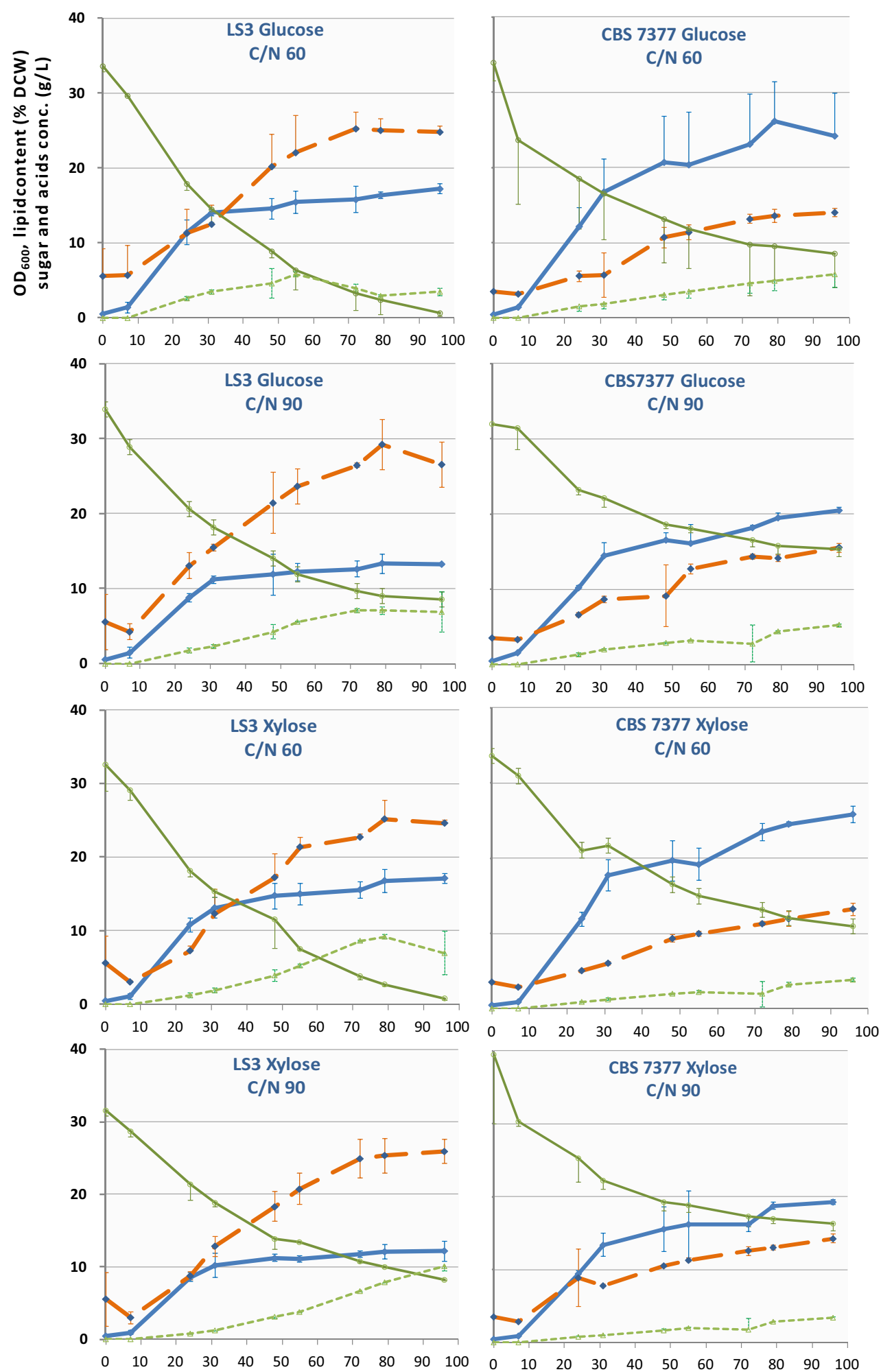

Fig. 3 Bioconversion of sugars into lipids during the growth of two strains of B. raffinosifermentans in nitrogen-limited YNB-based media. Glucose and $x y$ lose were each used as the $C$ source $(30 \mathrm{~g} / \mathrm{L})$ at two different $C / N$ ratios. Average values and the standard deviation $(n=3)$ for $O D_{600}(b l u e$ line), residual sugar in the medium ( $\mathrm{g} / \mathrm{L}$, plain green line), cumulative organic acids ( $\mathrm{g} / \mathrm{L}$, dotted green line), and lipid content (\% DCW, orange line) were plotted over time $(h)$ 

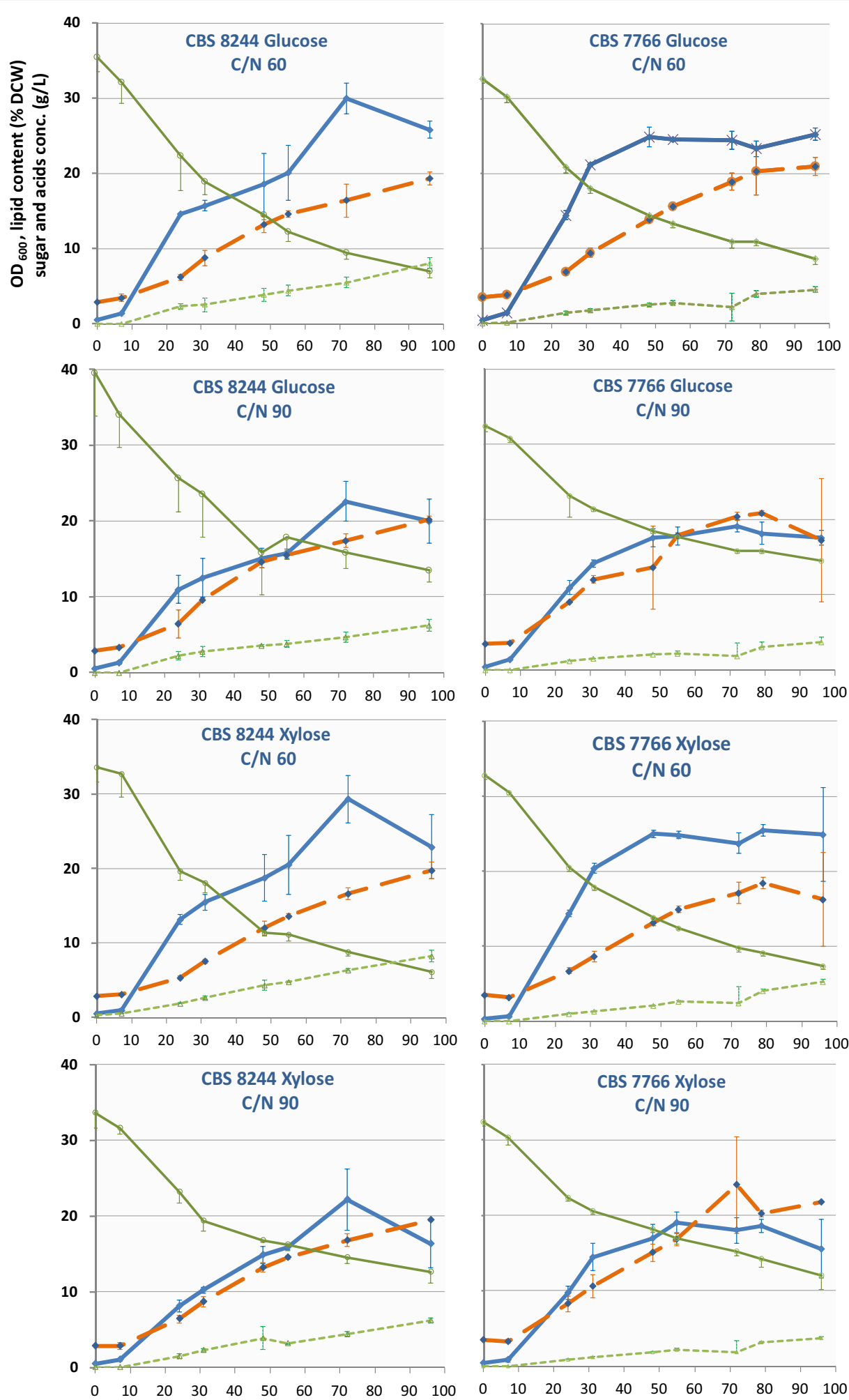

Fig. 4 Bioconversion of sugars to lipids during growth of two strains of B. adeninivorans in nitrogen-limited YNB-based media. Glucose and xylose were each used as the $C$ source $(30 \mathrm{~g} / \mathrm{L})$ at two different $C / N$ ratios. Average values and the standard deviation $(n=3)$ for $\mathrm{OD}_{600}$ (blue line), residual sugar in the medium ( $\mathrm{g} / \mathrm{L}$, plain green line), cumulative organic acids ( $\mathrm{g} / \mathrm{L}$, dotted green line), and lipid content (\% DCW, orange line) were plotted over time (h) 
adeninivorans CBS $8244^{\mathrm{T}}$ accumulated lipid more slowly and probably did not reach its maximum.

The excretion of organic compounds is a well-known competing sink for lipid production, particularly that of citric acid, both an intermediate of the TCA cycle and a precursor for acetyl-CoA in oleaginous yeasts. In our shake-flask experiments, we found two different organic acids to be excreted into the medium: citric acid and acetic acid. Globally, their cumulative concentration in the medium (Figs. 3,4 ) tended to steadily rise over time and reached $3.4 \pm 0.2 \mathrm{~g} / \mathrm{L}$ (CBS 7766 cultivated in xylose $\mathrm{C} / \mathrm{N}$ 90) to $10.1 \pm 0.6 \mathrm{~g} / \mathrm{L}$ (LS3 cultivated in xylose C/N 90) by the end of experiment. LS3 was unique concerning the excretion of organic acids, both in terms of their total concentration, which did not increase after 50 to $60 \mathrm{~h}$ of cultivation in some media, and the relative proportion of acetic to citric acid. Although acetic acid was the most abundant organic acid excreted by all other strains, excretions of citric acid was equivalent or more abundant in LS3 (Additional file 1: Figure S3). In glucose media, in particular, the final concentration of acetic acid was no more than half that of citric acid for this strain. This lower final concentration of acetic acid was due to reaching the plateau early (C/N 90, Additional file 1: Figure S3) or clear reuptake of this compound $(\mathrm{C} / \mathrm{N} 60$, Additional file 1: Figure S3), whereas glucose was not exhausted.

In terms of their FA composition, Blastobotrys strains synthesized and stored four main FAs during the $96 \mathrm{~h}$ of cultivation, accounting for more than $96 \%$ of total FA content: C18:1 (43 to 52\%, depending on the strain and medium), C16:0 (23 to 27\%), C18:2 (11 to 20\%), and C18:0 (4 to 11\%) (Additional file 1: Figure S4). C16 and C18 FAs are typically the most abundant FAs in a wide range of yeasts and fungi $[60,61]$. However, the relative proportion varies depending on the yeast and culture/ media conditions. The FA fraction was particularly poor in mono unsaturated C16:1. This feature was also noted for B. adeninivorans CBS $8244^{\mathrm{T}}$ in a comparative study of the FA composition of Saccharomycotina yeasts cultivated in YPD [62].

We found consistent but only very low levels of $\mathrm{C} 17$ chain-length FA $(<4 \mathrm{mg} / \mathrm{g} \mathrm{DCW})$. Indeed, yeast are not known to naturally synthesize large amounts of such odd-chain FAs, unless provided, for example, with oddcarbon precursors or benzoate [63-65]. Olstorpe et al. [17] reported FA profiles for CBS $8244^{\mathrm{T}}$ and CBS 7377 cultured in YPD medium, which were unexpectedly poor in oleic acid ( 3 to $11 \%$ of total FA at $30{ }^{\circ} \mathrm{C}$ ) and rich in C17:1 (20 to $29 \%$ of total FA at $30{ }^{\circ} \mathrm{C}$ ). The reason for these uncommon profiles is unknown, especially since Froissard et al. [62] reported the same four main FAs in B. adeninivorans CBS $8244^{\mathrm{T}}$ cultivated in YPD at $28^{\circ} \mathrm{C}$ as those observed here.

\section{Lipid synthesis from various carbon sources}

Sugars found in plants as reserve sugars or constituting lignocellulosic fibers, as well as the industrial by-product glycerol, are attractive substrates for microbial oil production. We tested the LS3 strain, our best lipid producer, for its ability to accumulate lipids while growing in various hexoses, polysaccharides, or glycerol. The FA fraction of the cells was characterized after 72 and $96 \mathrm{~h}$ of culture at $28{ }^{\circ} \mathrm{C}$ in YNB-based medium with the $\mathrm{C}$ source at $30 \mathrm{~g} / \mathrm{L}$ and $0.75 \mathrm{~g} / \mathrm{L} \mathrm{NH}_{4} \mathrm{Cl}$. After $96 \mathrm{~h}$, lipids accumulated to above $30 \% \mathrm{DCW}$ in all substrates, except galactose, starch, and glycerol (Fig. 5). Maximum lipid synthesis and storage was obtained for some of the sugars (i.e., cellobiose, glucose, sucrose, or fructose), likely rapidly assimilated, after $96 \mathrm{~h}$ in our shake-flask cultures since the rise between 72 and $96 \mathrm{~h}$ was less than 5.5\% (Additional file 2: Table S1). In contrast, glycerol appeared to be the least favorable substrate for bioconversion into lipids (total FAs reached only $16.1 \pm 0.4 \% \mathrm{DCW}$ ), probably because of its slow assimilation, which also affected the OD reached at $96 \mathrm{~h}: 11.4 \pm 1.0 \%$ in glycerol instead of $16.8 \pm 0.4 \%$ in cellobiose. We also observed small differences in the composition of FA profiles relative to that obtained in glucose, especially when LS3 was grown in starch or glycerol (Additional file 2: Table S1). Such variations that depend on the culture conditions have already been reported for other oleaginous yeasts [66]. In all cases, however, $\mathrm{C} 18: 1$ was the main FA, followed in decreasing proportion by $\mathrm{C} 16: 0, \mathrm{C} 18: 2$, and C18:0 (Additional file 2: Table S1). In conclusion, all tested substrates, from simple to complex, could be turned into lipids by strain LS3, although with varying efficiency.

\section{Efficient lipid synthesis at high temperature}

Blastobotrys adeninivorans and B. raffinosifermentans, represented by the strains listed in Table 1 , are known to grow at $45{ }^{\circ} \mathrm{C}$. Temperature is known to influence plasma membrane composition, which must adapt to preserve an adequate level of fluidity and function. The nature of FAs, including their chain length and degree of unsaturation (DUS), is involved in this aspect [67-69] and we reasoned that FAs may be stored or recycled accordingly. We thus investigated the ability of two strains, LS3 ( $B$. raffinosifermentans) and CBS $8244^{\mathrm{T}}$ (B. adeninivorans), to produce lipids at four different temperatures: 28,37 , 42 , and $45^{\circ} \mathrm{C}$.

Cultures in $\mathrm{C} / \mathrm{N} 60$ glucose media were periodically monitored by $\mathrm{OD}_{600}$ and microscopy to evaluate the temperature-dependent growth capacity of the strains and possible filamentation. Both strains grew at each temperature tested (Additional file 1: Figure S5). However, growth of LS3 was lower with high temperatures $\left(42{ }^{\circ} \mathrm{C}\right.$ and $45{ }^{\circ} \mathrm{C}$ ), in contrast to that of CBS $8244^{\mathrm{T}}$ (Additional 

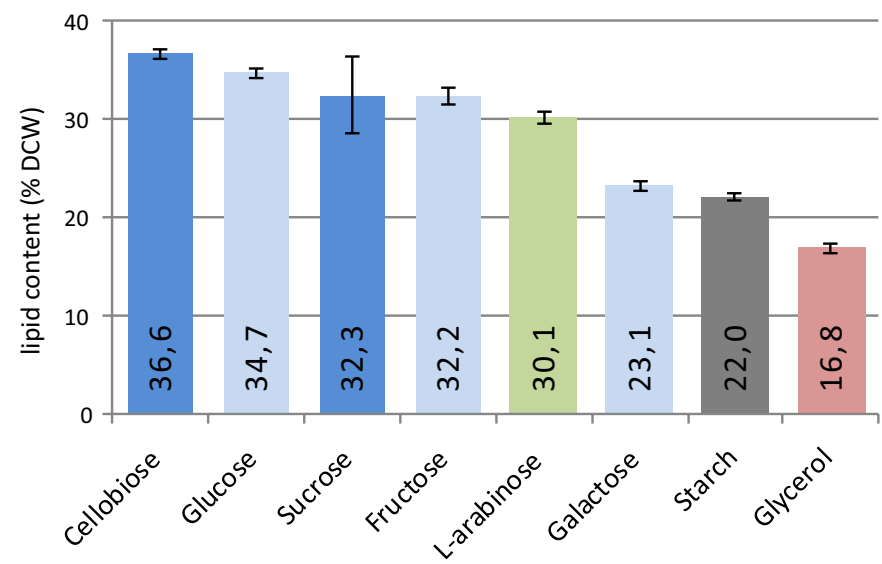

b
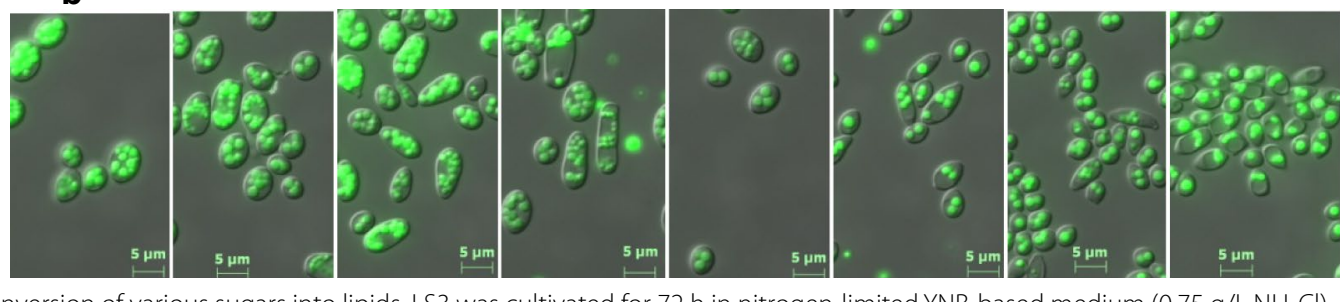

Fig. 5 Bioconversion of various sugars into lipids. LS3 was cultivated for $72 \mathrm{~h}$ in nitrogen-limited YNB-based medium $\left(0.75 \mathrm{~g} / \mathrm{L} \mathrm{NH}{ }_{4} \mathrm{Cl}\right)$ supplemented with the indicated sugars at $30 \mathrm{~g} / \mathrm{L}$. a Lipid content (\% DCW) is presented as a bar with the average value and mean deviation ( $n=2)$ and a color code indicating the nature of the carbon source: green, C5 monosaccharide; light blue, C6 monosaccharide; blue, disaccharide; gray, polysaccharide; red, polyol. b Microscopic images after BODIPY ${ }^{\circledR}$ staining of the $72 \mathrm{~h}$-cultivated cells in the relevant medium (same order as in the histograms)

file 1: Figure S5 for OD, Fig. 6a for biomass in $\mathrm{g} / \mathrm{L}$ ). Hyphae were only transiently detected in our lipogenic YNB medium (Additional file 1: Figure S5), contrary to other media, such as YPD, which gradually pushed the LS3 and CBS $8244^{\mathrm{T}}$ cells into (pseudo)hyphae phase above $37{ }^{\circ} \mathrm{C}$ and $42{ }^{\circ} \mathrm{C}$, respectively ([11], our unpublished results in YPD medium).

As observed for growth, the amount of total cellular FAs was independent of the temperature of cultivation for CBS $8244^{\mathrm{T}}$ : approximately $19 \%$ DCW (Fig. 6b). Instead, LS3 showed an optimum at $37{ }^{\circ} \mathrm{C}$ for lipid content, reaching 30\% DCW (approximately 20\% higher than at $28^{\circ} \mathrm{C}$ and $25 \%$ higher than at 42 to $45^{\circ} \mathrm{C}$ ). Despite the moderate decrease in lipid content of LS3 at temperatures above $37{ }^{\circ} \mathrm{C}$, we concluded that lipid metabolism of $B$. adeninivorans and $B$. raffinosifermentans was still active at temperatures of up to $45^{\circ} \mathrm{C}$.

Sugar consumption was temperature-dependent for both CBS $8244^{\mathrm{T}}$ and LS3, however, with opposite trends (Fig. 6c). For strain LS3, glucose consumption was lower at elevated temperatures $\left(42{ }^{\circ} \mathrm{C}\right.$ and $45^{\circ} \mathrm{C}$ ), consistent with the lower biomass and lipid content. Instead, glucose consumption of strain CBS $8244^{\mathrm{T}}$ was higher at elevated temperatures, though the amount of lipid and biomass were globally conserved. Overall, final bioconversion yields $\left(Y_{\mathrm{FA} / \mathrm{S}}\right)$ were within the range of 0.048 to
0.093 for LS3 and 0.056 to 0.070 for CBS $8244^{\mathrm{T}}$ (Fig. 6d and Additional file 2: Table S2). Higher yields were obtained at lower temperatures $\left(28^{\circ} \mathrm{C}, 37^{\circ} \mathrm{C}\right)$ for both strains; the difference was more pronounced, however, for LS3.

Finally, we examined the FA composition for each strain at each temperature in detail, early in the culture $(4.5 \mathrm{~h})$ and at $24 \mathrm{~h}$ and $72 \mathrm{~h}$ (Additional file 1: Figure S6 and Additional file 2: Table S3). The most divergent FA profiles between the two strains were globally observed at $4.5 \mathrm{~h}$. At that stage, as lipid content of the cells is still low (4 to $8 \% \mathrm{DCW}$ ), structural lipids are likely to significantly contribute to these differences. In this respect, a specific trait of CBS $8244^{\mathrm{T}}$ relative to LS3 is its richness in both $\mathrm{C} 16: 0$ and $\mathrm{C} 18: 2$, which is relatively independent of temperature (37 to $45^{\circ} \mathrm{C}$ ). A comparison of the proportions of FAs at 4.5 and $72 \mathrm{~h}$ showed that $\mathrm{C} 18: 1$ accumulated preferentially over C18:2 in CBS $8244^{\mathrm{T}}$, whereas C16:0 was preferentially stored over C18:0 in LS3. Overall, at $72 \mathrm{~h}$, the DUS of total FAs was slightly lower in CBS $8244^{\mathrm{T}}$ than in LS3, regardless of the temperature (by 6 to $14 \%)$.

Temperature had only a moderate effect on global FA composition of the cells. Indeed, we were unable to detect a specific temperature-dependent FA between 28 and $45^{\circ} \mathrm{C}$; the four main FAs remained the same and 


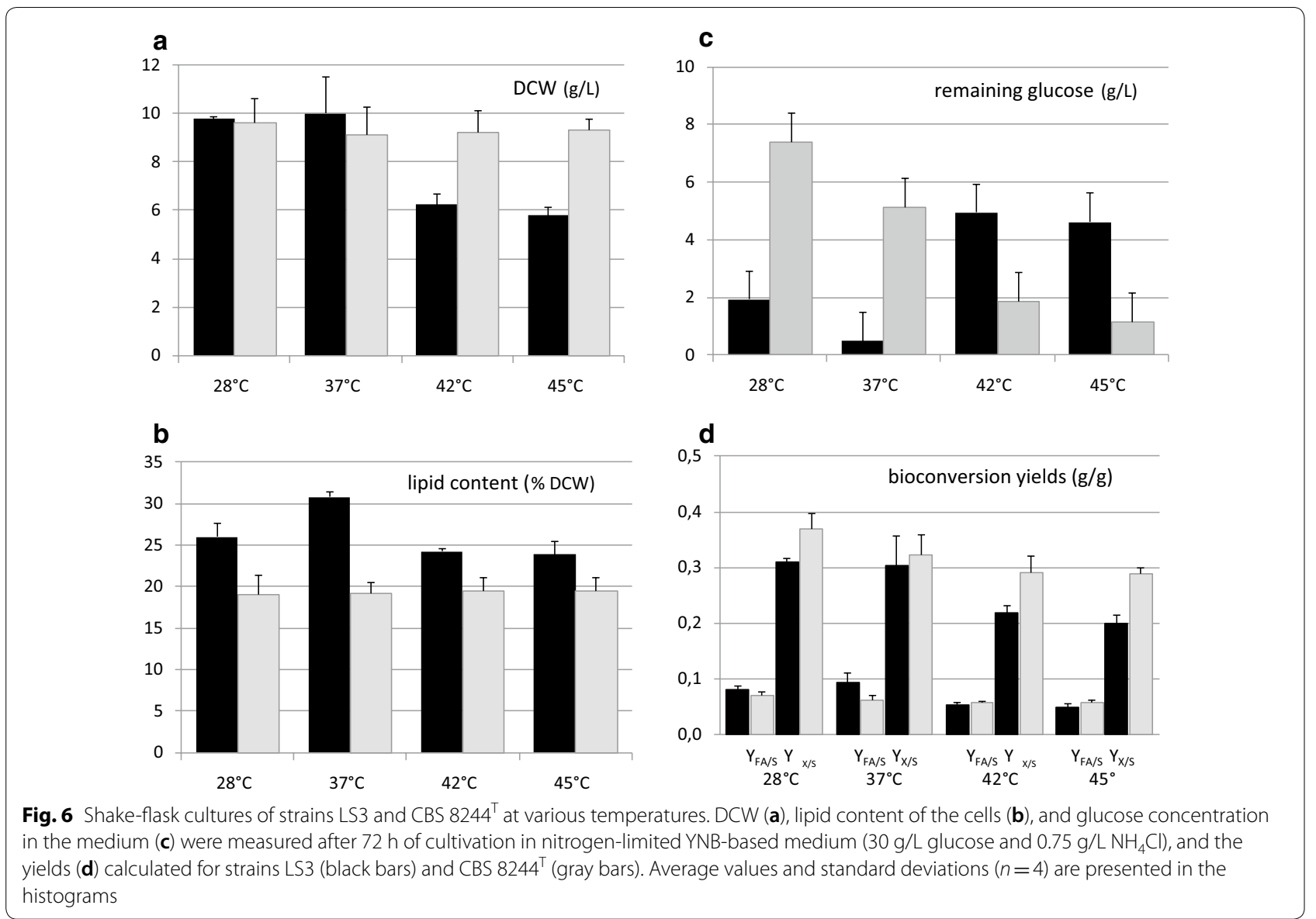

their relative abundance had only a moderate impact on the global DUS and chain-length index (C16/C18). The maximum temperature-dependent difference of the DUS was $8.5 \%$ and that of $\mathrm{C} 16 / \mathrm{C} 1838 \%$ for each strain at each stage of growth. Other authors have also observed that the major FAs in total lipids of various yeast species remained the same over a wide range of temperatures $[67,70,71]$, although the DUS and C16/C18 could be altered by more than $25 \%$ and $100 \%$, respectively [ 70 , 71]. Their detailed analysis revealed complex alterations in the abundance of FAs and its dependence on temperature, the specific nature of FAs depending on the species (in particular the presence of C18:3 or C16:1) and lipid class (i.e., different categories of phospholipids, triacylglycerols, etc.). In our analysis, which considered only total lipids, the alteration of FA composition, though modest, also appeared to be complex and strain and temperaturedependent. However, elevated temperatures had a similar effect for both CBS $8244^{\mathrm{T}}$ and LS3 for certain FAs at $72 \mathrm{~h}$ (Additional file 1: Figure S6). From 28 to $42^{\circ} \mathrm{C}, \mathrm{C} 16: 0$ and C18:1 decreased moderately in proportion to the total lipids, whereas C18:0 and C18:2 increased. Consequently, increasing temperatures led to more similar proportions of C16:0 and C18:2 (likely stored at this stage). The FA profile at $45{ }^{\circ} \mathrm{C}$ was highly similar to that at $42{ }^{\circ} \mathrm{C}$ for each strain. The strategy of adaptation to 'high' temperatures expectedly varies among species and strains; in the aforementioned $R$. toruloides thermotolerant mutant, a temperature increase from 30 to $37{ }^{\circ} \mathrm{C}$, while stimulating lipid production, drastically raised the relative proportion of C18:1, which reached $86 \%$ of total FA [32].

In conclusion, lipid metabolism of the LS3 and CBS $8244^{\mathrm{T}}$ strains was robust at temperatures from 28 to $45^{\circ} \mathrm{C}$. Temperature had a moderate impact on the final amount and nature of total cellular FAs, although LS3 showed optimal lipid production at $37^{\circ} \mathrm{C}$. Such robustness of lipid metabolism to differences in temperature is not shared across all yeasts. For example, the lipid content of $L$. starkeyi DSM 70295 reportedly decreased by $57 \%$ when the culture temperature was raised from 10 to $20{ }^{\circ} \mathrm{C}$ [70]. In the thermophilic species Kluyveromyces marxianus, 11 wild-type strains recently screened for lipogenesis (the best strain reportedly reached $10 \% \mathrm{DCW}$, mainly stored as free FAs) had a much lower FA content at 30 than $42^{\circ} \mathrm{C}$ [42]. 


\section{Conclusions}

Harnessing microbial lipid metabolism to produce oils or other chemicals of interest is a promising alternative to petroleum or plant oil. However, limitations in the use of yeast have been encountered, such as the poor utilization of certain types of substrates, weak xerotolerance, limited yields for lipid conversion in competition with other metabolic pathways, or difficult culturing in bioreactors (filamentation, foaming). We believe that the availability of a set of various oleaginous microorganisms adapted to different types of applications could be useful. Elevated temperatures may help reduce the costs of the process and participate in the solubilization of substrates. $B$. adeninivorans and B. raffinosifermentans exhibit many advantageous traits for biotechnological processes and, as reported here, were able to synthesize and store lipids at high temperatures, up to $45^{\circ} \mathrm{C}$.

Among four tested strains, LS3 was the best lipid-producing one. Although it showed a temperature dependence for growth, glucose consumption, and lipid storage, its native performance in the tested range of 28 to $45^{\circ} \mathrm{C}$ is a good starting point (24 to $30 \% \mathrm{DCW}$ from glucose).

As for other oleaginous yeasts, strain screening, process optimization, and/or metabolic engineering is likely to be successful. Knowledge of the lipid metabolism in these Blastobotrys species, as well as their genetic engineering, could be facilitated by their small genome. Accurate genome editing tools should be developed to complement existing classical genetic tools.

\section{Materials and methods}

\section{Strains, genotyping, identification}

Yeast strains formerly classified as $B$. adeninivorans are listed in Table 1; LS3 was in the collection of IPK, other strains were obtained from the CBS-KNAW. Y. lipolytica W29 was used as a control [72].

Genotyping of these strains was performed by intergenic spacer rDNA amplification and AluI Fingerprinting (IGSAF). The IGSAF technique has been successfully used to differentiate previously misidentified species in different taxa [73, 74].

The entire ITS + D1D2 region of the rDNA was amplified from genomic DNA in a single run, as previously described [75], using the primer pair ITSF (5' AGGAAC TAAAAGTCGTAACAAG) and DDR (5' GGTTTTACA CCCAAACACTC). After sequencing, the ITS and D1D2 sequences were delimited before multiple alignment with clustal X [76]. Sequences used for strain identification were as follows: ITS-D1D2 for LS3 was extracted from the LS3 genome sequence in the GRYC database [77] and mitochondrial COXII was retrieved from the NCBI accession number CBZY010000007; ITS-D1D2 fragments for CBS $8244^{\mathrm{T}}$, CBS 7350, CBS 7766, CBS 8335,
CBS 7377, CBS 7370, and mitochondrial mtCOXII (partial sequence) for CBS 7370 were deposited at the ENA under the study accession number PRJEB29673; type strain sequences for the mtCOXII marker were retrieved via YeastIP and the Genbank accession numbers were DQ443105.1 (CBS 6800 ${ }^{\mathrm{T}}$ ) and DQ443104.1 (CBS 8244).

\section{BLAST search}

Homologous gene products shared by Y. lipolytica E150 and $B$. raffinosifermentans LS3 were sought by reciprocal BLASTP with the BLOSUM62 matrix, using facilities of the GRYC database [77] and a threshold of $1 . \mathrm{e}^{-40}$.

\section{Media and growth conditions}

Rich medium YPD (yeast extract, peptone, and glucose, $10 \mathrm{~g} / \mathrm{L}$ each) was used for precultures and minimal medium (YNB) supplemented with various carbon sources for cultures. All YNB-based media contained $1.7 \mathrm{~g} / \mathrm{L}$ yeast nitrogen base (without amino acids or ammonium sulfate, Difco, Paris), $\mathrm{NH}_{4} \mathrm{Cl}$ as the nitrogen source (at various concentrations, as indicated), and were buffered with $50 \mathrm{mM}$ phosphate buffer $\left(\mathrm{Na}_{2} \mathrm{HPO}_{4}\right.$, $\mathrm{KH}_{2} \mathrm{PO}_{4}, \mathrm{pH}$ 6.8). Cells were cultured at $28{ }^{\circ} \mathrm{C}$ unless otherwise indicated. Absorbance at $600 \mathrm{~nm}\left(\mathrm{OD}_{600}\right)$ was measured with an LKB-Novaspec II spectrophotometer (Pharmacia), except for growth tests in microplates.

Growth tests were carried out in 96-well microplates (Greiner) incubated in a BIOTEK synergy MX plate reader under strong and constant agitation. $\mathrm{OD}_{600}$ was measured every $20 \mathrm{~min}$. Wells were filled with $190 \mu \mathrm{L}$ YNB-based buffered media with glucose, xylose, or glycerol (at either 10 or $2 \mathrm{~g} / \mathrm{L}$ ), all supplemented with $5 \mathrm{~g} / \mathrm{L}$ $\mathrm{NH}_{4} \mathrm{CL}$. They were inoculated with $10 \mu \mathrm{L}$ of a cell suspension from overnight precultures in YPD and adjusted to an $\mathrm{OD}_{600}$ of 4 by the addition of the desired volume of YNB (without carbon) to the cell pellet.

\section{Cultures for lipid accumulation}

Experiments were carried out in $500-\mathrm{mL}$ baffled flasks filled with $100 \mathrm{~mL}$ YNB-based medium and incubated under agitation at $160 \mathrm{rpm}$ in a Minitron incubator (INFOR-HT, Swisserland). Carbon sources were added at a concentration of $30 \mathrm{~g} / \mathrm{L}$. When indicated, the carbonto-nitrogen mass ratio $(\mathrm{C} / \mathrm{N})$ was adjusted to 60 or 90 by adding $0.75 \mathrm{~g} / \mathrm{L}$ or $0.5 \mathrm{~g} / \mathrm{L} \mathrm{NH}_{4} \mathrm{Cl}$, respectively. Oleic acid (65.0-88.0\%, Sigma-Aldrich) used in this study contained 70\% C18:1 $(n-9)$ cis, 3\% C18:1 $(n-9)$ trans, 7\% C18:2 $(n-6), 1.2 \%$ C17:1, 3.9\% C16:0, 4.7\% C16:1 (n - 7), 0.9\% C16:1 $(n-9)$, and other FAs in low amounts [78]. Cultures were routinely performed at $28{ }^{\circ} \mathrm{C}$. The experiment testing various temperatures was carried out for $72 \mathrm{~h}$ in four incubators in which the temperature was recorded by USB thermometers. 
Precultures were grown for $20 \mathrm{~h}$ at $28^{\circ} \mathrm{C}$ in flasks filled with YPD and used for culture inoculation at an $\mathrm{OD}_{600 \mathrm{~nm}}$ of 0.5. YPD of the preculture was eliminated by centrifugation and the cells resuspended in YNB without carbon and nitrogen.

\section{Lipid and biomass quantification}

Lipid content of the cells was determined by quantifying their FA fraction, as previously described [59]. Cells from-10 mL culture samples were collected in pre-weighed tubes, washed twice in water (for cultures in sugar-based medium), or twice successively in a mixture of $0.5 \% \mathrm{BSA}$ and $0.9 \% \mathrm{NaCl}$ and once in water (for oily medium), and resuspended in $1 \mathrm{~mL}$ water. After freeze-drying for $24 \mathrm{~h}$ at $-55{ }^{\circ} \mathrm{C}$ (Alpha 1-2Dplus, Bioblock Scientific), samples in their tubes were weighed and stored at $-20{ }^{\circ} \mathrm{C}$. The difference in tube weights represented the mass of the cells found in $10-\mathrm{mL}$ cultures and permitted calculation of the biomass in $\mathrm{g} / \mathrm{L}$.

Fatty acid methyl esters (FAMES) were recovered from 10 to $20 \mathrm{mg}$ aliquots of freeze-dried cells ( 2 to $4 \mathrm{mg}$ in samples collected at $4.5 \mathrm{~h}$ ), using a hot methanol- $\mathrm{H}_{2} \mathrm{SO}_{4}$ method, adapted from Browse et al. [79], and analyzed by gas chromatography on a Varian 430 equipped with a flame-ionization detector and a FactorFour vf- $23 \mathrm{~ms}$ column. FAMES were identified by comparison to commercial standards (FAME32; Supelco); an internal FA standard (100 $\mu \mathrm{g}$ C12:0 from Sigma) was added to each sample prior to transesterification to enable FA quantification in the analyzed aliquots.

Lipid content is expressed with respect to DCW: lipid content of $1 \% \mathrm{DCW}=10 \mathrm{mg}$ FA per $\mathrm{g}$ of dry cell.

\section{FA profiles, index, statistics}

The main identified FAs were: C18:1 $(n-9)$ cis (oleic acid, simplified as C18:1 in the text), C18:0 (stearic acid), C18:2 $(n-6)$ cis (linoleic acid, simplified as C18:2 in the text), and C16:0 (palmitic acid). C16:1 $(n-9)$ cis (palmitoleic acid) and C16:1 $(n-7)$, both grouped under C16:1, were also identified, in low amounts. Unidentified or minor FAs were grouped under 'other FAs'; among them, C18:3 was sporadically detected (relative amount $<0.45 \%$ ).

The DUS [69] was calculated as follows: (percentage of monoene FAs $+2 \times$ the percentage of diene FAs)/total percentage of four main FAs. C16/C18 [70] was calculated as the ratio of carbon-chain length of 16 to carbonchain length of 18 for the four main FAs.

Kruskal and Wallis tests with multiple comparisons [80] were run using Excel facilities designed by P. Georgin, M. Gouet, and G. Le Pape.

\section{Quantification of sugars and organic acids}

Sugars and organic acids in the culture supernatants were analyzed by the HPLC method of calibration with external standards. Immediately after centrifugation of culture samples, supernatants were conserved frozen $\left(-20{ }^{\circ} \mathrm{C}\right)$. Prior to analysis, they were homogenized, centrifuged again to eliminate any remaining cells, and diluted 10 times in water. They were analyzed by HPLC (UltiMate 3000, Dionex-ThermoFisher Scientific, UK) coupled to UV (210 nm) and RI detectors. The Aminex HPX87H column (Thermo Scientific, Waltham, MA) was eluted with $0.01 \mathrm{~N} \mathrm{H}_{2} \mathrm{SO}_{4}$ at $35^{\circ} \mathrm{C}$ at a flow rate of $0.6 \mathrm{~mL} / \mathrm{min}$. Peak integration, identification, and quantification were performed using CHROMELEON software (Thermo Scientific, USA) and standards purchased at Sigma-Aldrich.

\section{Cell imaging}

Images were acquired using a Zeiss Axio Imager M2 microscope (Zeiss, Le Pecq, France) fitted with an Apochromat $100 \times / 1.40$ oil M27 objective and operated with Axiovision 4.8.2 software. Fluorescently-stained lipid bodies were visualized using Zeiss filters 45 and 46, after incubating culture samples with $1.5 \mu \mathrm{g} / \mathrm{mL}$ BODIPY $^{\circledR}$ Lipid Probe (493/503 (D-3922), Invitrogen) for at least $15 \mathrm{~min}$ at room temperature.

\section{Additional files}

\begin{abstract}
Additional file 1: Figure S1. Polymorphisms in the ITS-D1D2 nucleotide sequence. Figure S2. Growth of B. adeninivorans and B. raffinosifermentans in three different substrates. Figure S3. Organic acids excreted in lipogenic culture conditions. Figure S4. FA composition of strains of $B$. raffinosifermentans and $B$. adeninivorans after growth in lipogenic medium. Figure S5. Growth curves and microscopic images of strains LS3 and CBS $8244^{\top}$ cultured in glucose lipogenic medium at various temperatures. Figure S6. Relative FA composition of strains LS3 and CBS $8244^{\top}$ at various times and temperatures of cultivation in glucose lipogenic medium.

Additional file 2: Table S1. Relative composition (\%) of the FA fraction of LS3 cells after growth in various substrates. Table S2. Final yields after $72 \mathrm{~h}$ of cultivation of LS3 and CBS8244 ${ }^{\top}$ in YNB-based glucose media $(C / N=60)$ at different temperatures. Table S3. Time course analysis of the FA profiles of strains LS3 and CBS $8244^{\top}$ cultivated in YNB-based glucose media $(\mathrm{C} / \mathrm{N}=60)$ at various temperatures.
\end{abstract}

\section{Acknowledgements}

We thank A. Beopoulos, R. Ledesma-Amaro, and J-M. Nicaud for helpful discussions, and R. Ledesma-Amaro for critical reading of the manuscript. We also thank William Hempel of Alex Edelman and Associates for English editing of the manuscript.

\section{Authors' contributions}

CN and AMCLC conceived and supervised the study. ST and DS performed experiments for lipid production and participated in their analysis. FF performed preliminary experiments for lipid production and carried out growth tests with AMCLC. VN performed IGSAF and genotyping. GK provided strain LS3 and knowledge on the species and discussed the results. AMCLC wrote the manuscript, which was reviewed and commented on by all authors. All authors read and approved the final manuscript. 


\section{Funding}

This work was supported by INRA; DS was funded by IsDB (Islamic Development Bank) during his Ph.D.

\section{Availability of data and materials}

All data generated or analyzed during this study are included in this published article and its additional files.

\section{Ethics approval and consent to participate}

Not applicable.

\section{Consent for publication}

Not applicable.

\section{Competing interests}

The authors declare that they have no competing interests.

\section{Author details}

${ }^{1}$ Micalis Institute, INRA, AgroParisTech, Université Paris-Saclay, 78350 Jouy-en-Josas, France. ${ }^{2}$ Leibniz Institute of Plant Genetics and Crop Plant Research (IPK), Correnstr. 3, 06466 Gatersleben, Germany.

Received: 4 December 2018 Accepted: 9 June 2019

Published online: 20 June 2019

\section{References}

1. Kurtzman CP, Robnett CJ. Multigene phylogenetic analysis of the Trichomonascus, Wickerhamiella and Zygoascus yeast clades, and the proposal of Sugiyamaella gen. nov. and 14 new species combinations. FEMS Yeast Res. 2007;7:141-51.

2. Middelhoven WJ, Hoogkamer-Te Niet MC, Kreger-Van Rij NJ. Trichosporon adeninovorans sp. nov., a yeast species utilizing adenine, xanthine, uric acid, putrescine and primary n-alkylamines as the sole source of carbon, nitrogen and energy. Antonie Van Leeuwenhoek. 1984;50:369-78.

3. Van der Walt JP, Smith MT, Yamada Y. Arxula gen. nov. (Candidaceae), a new anamorphic, arthroconidial yeast genus. Antonie Van Leeuwenhoek. 1990;57:59-61.

4. Samsonova IA, Kunze G, Bode R, Bottcher F. A set of genetic markers for the chromosomes of the imperfect yeast Arxula adeninivorans. Yeast. 1996:12:1209-17.

5. Rosel H, Kunze G. Integrative transformation of the dimorphic yeast Arxula adeninivorans LS3 based on hygromycin B resistance. Curr Genet. 1998;33:157-63.

6. Middelhoven WJ, de Jong IM, de Winter M. Arxula adeninivorans, a yeast assimilating many nitrogenous and aromatic compounds. Antonie Van Leeuwenhoek. 1991;59:129-37.

7. Buttner R, Bode R, Birbaum D. Alcoholic fermentation of starch by Arxula adeninivorans. Zentralbl Mikrobiol. 1992;147:225-30.

8. Boer E, Schroter A, Bode R, Piontek M, Kunze G. Characterization and expression analysis of a gene cluster for nitrate assimilation from the yeast Arxula adeninivorans. Yeast. 2009;26:83-93.

9. Robert V, Cardinali G, Casadevall A. Distribution and impact of yeast thermal tolerance permissive for mammalian infection. BMC Biol. 2015;13:18.

10. Radecka D, Mukherjee V, Mateo RQ, Stojiljkovic M, Foulquie-Moreno MR, Thevelein JM. Looking beyond Saccharomyces: the potential of nonconventional yeast species for desirable traits in bioethanol fermentation. FEMS Yeast Res. 2015;15:fov053.

11. Wartmann T, Kruger A, Adler K, Duc BM, Kunze I, Kunze G. Temperaturedependent dimorphism of the yeast Arxula adeninivorans Ls3. Antonie Van Leeuwenhoek. 1995;68:215-23.

12. Bischoff F, Chamas A, Litwinska K, Matthes F, Böer E, Kunze G. Application of Blastobotrys (Arxula) adeninivorans in biotechnology. In: Satyanarayana T, Kunze G, editors. Yeast diversity in human welfare. Singapore: Springer Singapore; 2017. p. 455-79.

13. Kunze G, Gaillardin C, Czernicka M, Durrens P, Martin T, Boer E, et al. The complete genome of Blastobotrys (Arxula) adeninivorans LS3 - a yeast of biotechnological interest. Biotechnol Biofuels. 2014;7:66.
14. Malak A, Baronian K, Kunze G. Blastobotrys (Arxula) adeninivorans: a promising alternative yeast for biotechnology and basic research. Yeast. 2016:33:535-47.

15. Stockmann C, Scheidle M, Dittrich B, Merckelbach A, Hehmann G, Melmer $\mathrm{G}$, et al. Process development in Hansenula polymorpha and Arxula adeninivorans, a re-assessment. Microb Cell Fact. 2009;8:22.

16. Maskow T, Rollich A, Fetzer I, Ackermann JU, Harms H. On-line monitoring of lipid storage in yeasts using impedance spectroscopy. J Biotechnol. 2008; 135:64-70.

17. Olstorpe M, Pickova J, Kiessling A, Passoth V. Strain- and temperaturedependent changes of fatty acid composition in Wickerhamomyces anomalus and Blastobotrys adeninivorans. Biotechnol Appl Biochem. 2014:61:45-50.

18. Adrio JL. Oleaginous yeasts: promising platforms for the production of oleochemicals and biofuels. Biotechnol Bioeng. 2017;114:1915-20.

19. Dujon B, Sherman D, Fischer G, Durrens P, Casaregola S, Lafontaine I, et al. Genome evolution in yeasts. Nature. 2004;430:35-44.

20. Kumar S, Kushwaha H, Bachhawat AK, Raghava GP, Ganesan K. Genome sequence of the oleaginous red yeast Rhodosporidium toruloides MTCC 457. Eukaryot Cell. 2012;11:1083-4.

21. Riley R, Haridas S, Wolfe KH, Lopes MR, Hittinger CT, Goker M, et al. Comparative genomics of biotechnologically important yeasts. Proc Natl Acad Sci USA. 2016;113:9882-7

22. McNeil BA, Stuart DT. Lipomyces starkeyi: an emerging cell factory for production of lipids, oleochemicals and biotechnology applications. World J Microbiol Biotechnol. 2018;34:147.

23. Sutanto S, Zullaikah S, Tran-Nguyen PL, Ismadji S, Ju Y-H. Lipomyces starkeyi: its current status as a potential oil producer. Fuel Process Technol. 2018;177:39-55.

24. Park Y-K, Nicaud J-M, Ledesma-Amaro R. The engineering potential of Rhodosporidium toruloides as a workhorse for biotechnological applications. Trends Biotechnol. 2018;36:304-17.

25. Gong Z, Wang Q, Shen H, Hu C, Jin G, Zhao ZK. Co-fermentation of cellobiose and xylose by Lipomyces starkeyi for lipid production. Bioresour Technol. 2012;117:20-4.

26. Papanikolaou S, Rontou M, Belka A, Athenaki M, Gardeli C, Mallouchos A, et al. Conversion of biodiesel-derived glycerol into biotechnological products of industrial significance by yeast and fungal strains. Eng Life Sci. 2017;17:262-81.

27. Hittinger CT, Rokas A, Bai FY, Boekhout T, Goncalves P, Jeffries TW, et al. Genomics and the making of yeast biodiversity. Curr Opin Genet Dev. 2015;35:100-9.

28. Michely S, Gaillardin C, Nicaud JM, Neuveglise C. Comparative physiology of oleaginous species from the Yarrowia clade. PLoS ONE. 2013;8:e63356.

29. Tai M, Stephanopoulos G. Engineering the push and pull of lipid biosynthesis in oleaginous yeast Yarrowia lipolytica for biofuel production. Metab Eng. 2013;15:1-9.

30. Timoumi A, Guillouet SE, Molina-Jouve C, Fillaudeau L, Gorret N. Impacts of environmental conditions on product formation and morphology of Yarrowia lipolytica. Appl Microbiol Biotechnol. 2018;102:3831-48.

31. Rakicka M, Lazar Z, Dulermo T, Fickers P, Nicaud JM. Lipid production by the oleaginous yeast Yarrowia lipolytica using industrial by-products under different culture conditions. Biotechnol Biofuels. 2015:8:104.

32. Wu C-C, Tsai Y-Y, Ohashi T, Misaki R, Limtong S, Fujiyama K. Isolation of a thermotolerant Rhodosporidium toruloides DMKU3-TK16 mutant and its fatty acid profile at high temperature. FEMS Microbiol Lett. 2018;365:fny203.

33. Abdel-Banat BMA, Hoshida H, Ano A, Nonklang S, Akada R. Hightemperature fermentation: how can processes for ethanol production at high temperatures become superior to the traditional process using mesophilic yeast? Appl Microbiol Biotechnol. 2010;85:861-7.

34. Guo Z, Duquesne S, Bozonnet S, Nicaud J-M, Marty A, O'Donohue MJ. Expressing accessory proteins in cellulolytic Yarrowia lipolytica to improve the conversion yield of recalcitrant cellulose. Biotechnol Biofuels. 2017;10:298.

35. Tansey MR, Brock TD. The upper temperature limit for eukaryotic organisms. Proc Natl Acad Sci USA. 1972;69:2426-8.

36. Mouchacca J. Heat tolerant fungi and applied research: addition to the previously treated group of strictly thermotolerant species. World J Microbiol Biotechnol. 2007;23:1755.

37. de Oliveira TB, Gomes E, Rodrigues A. Thermophilic fungi in the new age of fungal taxonomy. Extremophiles. 2015;19:31-7. 
38. Lobs A-K, Schwartz C, Wheeldon I. Genome and metabolic engineering in non-conventional yeasts: current advances and applications. Synth Syst Biotechnol. 2017;2:198-207.

39. Polburee P, Yongmanitchai W, Lertwattanasakul N, Ohashi T, Fujiyama K, Limtong S. Characterization of oleaginous yeasts accumulating high levels of lipid when cultivated in glycerol and their potential for lipid production from biodiesel-derived crude glycerol. Fungal Biol. 2015;119:1194-204.

40. Sitepu IR, Sestric R, Ignatia L, Levin D, German JB, Gillies LA, et al. Manipulation of culture conditions alters lipid content and fatty acid profiles of a wide variety of known and new oleaginous yeast species. Bioresour Technol. 2013;144:360-9.

41. Gientka I, Kieliszek M, Jermacz K, Blazejak S. Identification and characterization of oleaginous yeast isolated from kefir and its ability to accumulate intracellular fats in deproteinated potato wastewater with different carbon sources. Biomed Res Int. 2017;2017:6061042.

42. Cernak P, Estrela R, Poddar S, Skerker JM, Cheng Y-F, Carlson AK, et al. Engineering Kluyveromyces marxianus as a robust synthetic biology platform host. mBio. 2018;9:e01410-8.

43. Beopoulos A, Cescut J, Haddouche R, Uribelarrea JL, Molina-Jouve C, Nicaud JM. Yarrowia lipolytica as a model for bio-oil production. Prog Lipid Res. 2009;48:375-87.

44. Ratledge C. Regulation of lipid accumulation in oleaginous micro-organisms. Biochem Soc Trans. 2002;30:1047-50.

45. Beopoulos A, Nicaud JM, Gaillardin C. An overview of lipid metabolism in yeasts and its impact on biotechnological processes. Appl Microbiol Biotechnol. 2011;90:1193-206.

46. Boulton CA, Ratledge C. Correlation of lipid accumulation in yeasts with possession of ATP:citrate lyase. J Gen Microbiol. 1981;127:169-76.

47. Vorapreeda T, Thammarongtham C, Cheevadhanarak S, Laoteng K. Alternative routes of acetyl-CoA synthesis identified by comparative genomic analysis: involvement in the lipid production of oleaginous yeast and fungi. Microbiology. 2012;158:217-28.

48. Dulermo R, Gamboa-Melendez H, Ledesma-Amaro R, Thevenieau F, Nicaud JM. Yarrowia lipolytica AAL genes are involved in peroxisomal fatty acid activation. Biochim Biophys Acta. 2016;1861:555-65.

49. Liang MH, Jiang JG. Advancing oleaginous microorganisms to produce lipid via metabolic engineering technology. Prog Lipid Res. 2013;52:395-408.

50. Athenstaedt K. YALIOE32769g (DGA1) and YALIOE16797g (LRO1) encode major triacylglycerol synthases of the oleaginous yeast Yarrowia lipolytica. Biochim Biophys Acta. 2011;1811:587-96.

51. Beopoulos A, Haddouche R, Kabran P, Dulermo T, Chardot T, Nicaud JM. Identification and characterization of DGA2, an acyltransferase of the DGAT1 acyl-CoA:diacylglycerol acyltransferase family in the oleaginous yeast Yarrowia lipolytica. New insights into the storage lipid metabolism of oleaginous yeasts. Appl Microbiol Biotechnol. 2012;93:1523-37.

52. Zhang H, Damude HG, Yadav NS. Three diacylglycerol acyltransferases contribute to oil biosynthesis and normal growth in Yarrowia lipolytica. Yeast Chichester Engl. 2012;29:25-38.

53. Brevnova EE, Shaw AJ, Van Diken JP. Increasing lipid production and optimizing lipid composition. Cambridge: Novogy, Inc:; 2015.

54. Kunze G, Kunze I. Characterization of Arxula adeninivorans strains from different habitats. Antonie Van Leeuwenhoek. 1994;65:29-34.

55. Weiss S, Samson F, Navarro D, Casaregola S. YeastIP: a database for identification and phylogeny of Saccharomycotina yeasts. FEMS Yeast Res. 2013;13:117-25.

56. Klein M, Swinnen S, Thevelein JM, Nevoigt E. Glycerol metabolism and transport in yeast and fungi: established knowledge and ambiguities. Environ Microbiol. 2017;19:878-93.

57. Workman M, Holt P, Thykaer J. Comparing cellular performance of Yarrowia lipolytica during growth on glucose and glycerol in submerged cultivations. AMB Express. 2013;3:58.

58. Bommareddy RR, Sabra W, Maheshwari G, Zeng A-P. Metabolic network analysis and experimental study of lipid production in Rhodosporidium toruloides grown on single and mixed substrates. Microb Cell Fact. 2015;14:36.

59. Ledesma-Amaro R, Lazar Z, Rakicka M, Guo Z, Fouchard F, Crutz-Le Coq A-M, et al. Metabolic engineering of Yarrowia lipolytica to produce chemicals and fuels from xylose. Metab Eng. 2016;38:115-24.

60. Bharathiraja B, Sridharan S, Sowmya V, Yuvaraj D, Praveenkumar R. Microbial oil—a plausible alternate resource for food and fuel application. Bioresour Technol. 2017:233:423-32.
61. Li Q, Du W, Liu D. Perspectives of microbial oils for biodiesel production. Appl Microbiol Biotechnol. 2008;80:749-56.

62. Froissard M, Canonge M, Pouteaux M, Cintrat B, Mohand-Oumoussa S, Guillouet SE, et al. Lipids containing medium-chain fatty acids are specific to post-whole genome duplication Saccharomycotina yeasts. BMC Evol Biol. 2015;15:97.

63. Amara S, Seghezzi N, Otani H, Diaz-Salazar C, Liu J, Eltis LD. Characterization of key triacylglycerol biosynthesis processes in rhodococci. Sci Rep. 2016;6:24985.

64. Matatkova O, Gharwalova L, Zimola M, Rezanka T, Masak J, Kolouchova I. Using odd-alkanes as a carbon source to increase the content of nutritionally important fatty acids in Candida krusei, Trichosporon cutaneum, and Yarrowia lipolytica. Int J Anal Chem. 2017;2017:8195329.

65. Rezanka T, Kolouchova I, Sigler K. Precursor directed biosynthesis of oddnumbered fatty acids by different yeasts. Folia Microbiol. 2015;60:457-64.

66. Yun EJ, Lee J, Kim DH, Kim J, Kim S, Jin Y-S, et al. Metabolomic elucidation of the effects of media and carbon sources on fatty acid production by Yarrowia lipolytica. J Biotechnol. 2018;272-273:7-13.

67. Suutari M, Liukkonen $\mathrm{K}$, Laakso S. Temperature adaptation in yeasts: the role of fatty acids. J Gen Microbiol. 1990;136:1469-74.

68. Rossi M, Buzzini P, Cordisco L, Amaretti A, Sala M, Raimondi S, et al. Growth, lipid accumulation, and fatty acid composition in obligate psychrophilic, facultative psychrophilic, and mesophilic yeasts. FEMS Microbiol Ecol. 2009;69:363-72.

69. Arthur H, Watson K. Thermal adaptation in yeast: growth temperatures, membrane lipid, and cytochrome composition of psychrophilic, mesophilic, and thermophilic yeasts. J Bacteriol. 1976;1 28:56-68.

70. Suutari M, Rintamaki A, Laakso S. The effect of temperature on lipid classes and their fatty acid profiles in Lipomyces starkeyi. J Am Oil Chem Soc. 1996;73:1071-3.

71. Suutari M, Rintamaki A, Laakso S. Membrane phospholipids in temperature adaptation of Candida utilis: alterations in fatty acid chain length and unsaturation. J Lipid Res. 1997;38:790-4.

72. Lazar Z, Dulermo T, Neuveglise C, Crutz-Le Coq AM, Nicaud JM. Hexokinase-a limiting factor in lipid production from fructose in Yarrowia lipolytica. Metab Eng. 2014;26:89-99.

73. Nguyen H-V, Gaillardin C, Neuveglise C. Differentiation of Debaryomyces hansenii and Candida famata by rRNA gene intergenic spacer fingerprinting and reassessment of phylogenetic relationships among $D$. hansenii, $C$. famata, D. fabryi, C. flareri (= D. subglobosus) and D. prosopidis: description of $D$. vietnamensis sp. nov. closely related to D. nepalensis. FEMS Yeast Res. 2009;9:641-62

74. Cornet M, Sendid B, Fradin C, Gaillardin C, Poulain D, Nguyen H-V. Molecular identification of closely related Candida species using two ribosomal intergenic spacer fingerprinting methods. J Mol Diagn JMD. 2011;13:12-22.

75. Nguyen $\mathrm{H}-\mathrm{V}$, Boekhout T. Characterization of Saccharomyces uvarum (Beijerinck, 1898) and related hybrids: assessment of molecular markers that predict the parent and hybrid genomes and a proposal to name yeast hybrids. FEMS Yeast Res. 2017;17:fox014.

76. Larkin MA, Blackshields G, Brown NP, Chenna R, McGettigan PA, McWilliam H et al. Clustal W and Clustal X version 2.0. Bioinformatics. 2007;23:2947-8.

77. Neuvéglise C, Devillers H. Genome resources for yeast chromosomes. http://gryc.inra.fr. Accessed 9 May 2019.

78. Dulermo R, Gamboa-Melendez H, Dulermo T, Thevenieau F, Nicaud $J M$. The fatty acid transport protein Fat $1 p$ is involved in the export of fatty acids from lipid bodies in Yarrowia lipolytica. FEMS Yeast Res. 2014;14:883-96.

79. Browse J, McCourt PJ, Somerville CR. Fatty acid composition of leaf lipids determined after combined digestion and fatty acid methyl ester formation from fresh tissue. Anal Biochem. 1986;152:141-5.

80. Siegel S, Castellan NJ. Nonparametric statistics for the behavioral science. 2nd ed. New York: McGraw-Hill Inc.; 1988.

\section{Publisher's Note}

Springer Nature remains neutral with regard to jurisdictional claims in published maps and institutional affiliations. 